\title{
Role Entity
}

National Cancer Institute

\section{Source}

National Cancer Institute. Role Entity. NCI Thesaurus. Code C42754.

A non-hierarchical, named relationship between concepts. They are binary relationships pointing from one concept to another concept. Roles are unidirectional and each role has a Domain and a Range; the role points from one kind, the domain, to another (or same) kind, the range. The specific concept pointed to is considered the Role Value. 